
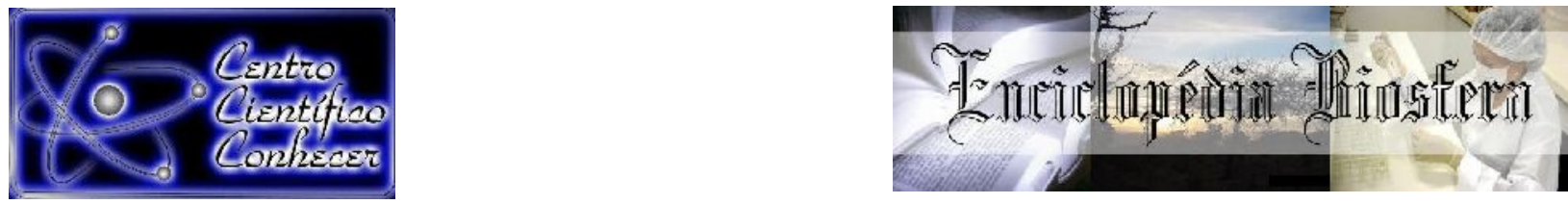

\title{
ASPECTOS CLÍNICOS E SOCIAIS DOS PORTADORES DA DOENÇA DE ALZHEIMER NA CIDADE DE MURITIBA-BA
}

Taiane Lopes Magalhães ${ }^{1}$, Belayrla Cerqueira de Jesus ${ }^{2}$, Lourenço Luis Botelho de Santana $^{3}$

${ }^{1}$ Bacharel em Farmácia da Faculdade Maria Milza (FAMAM), Governador Mangabeira - BA, Brasil

${ }^{2}$ Mestranda do Programa de Pós-Graduação em Enfermagem e SaúdeUniversidade Federal da Bahia (UFBA), Salvador-BA, Brasil.

${ }^{3}$ Professor Doutor dos cursos de Farmácia da Faculdade Maria Milza (FAMAM), Governador Mangabeira- BA, Brasil e Universidade do Estado da Bahia(UNEB), Departamento de Ciências da Vida, Salvador-BA, Brasil. Email: lourencoluisbotelho@gmail.com

\section{Recebido em: 15/08/2020 - Aprovado em: 15/09/2020 - Publicado em: 30/09/2020} DOI: 10.18677/EnciBio_2020C9

\begin{abstract}
A doença de Alzheimer acomete frequentemente idosos, afetando funções cognitivas como a memória, aprendizado, linguagem, atenção, habilidade visual e noção espacial. Na cidade de Muritiba-BA, nota-se um aumento dos casos da doença de Alzheimer, desta forma, foi necessário o aprofundamento do tema na cidade a fim de melhorar a qualidade de vida dos pacientes. O presente trabalho teve como objetivo principal analisar o perfil dos pacientes portadores da Doença de Alzheimer no município de Muritiba- BA no ano de 2019. Trata-se de um estudo descritivo de corte transversal, tendo como população os pacientes com a doença de Alzheimer da cidade de Muritiba-BA, cadastrados na atenção básica do munícipio. Verificou-se maior prevalência de idosos com Alzheimer entre o sexo feminino, de idade mais avançada, de baixa escolaridade e renda, e que eram cuidadas, geralmente, pela filha. A maioria dos idosos faziam uso de quatro ou mais medicamentos, sendo a quetiapina e a donepezila os mais utilizados. Os pacientes em sua maioria fazem uso de mais de 4 medicamentos diários no combate à doença e outras enfermidades. Desta forma, este trabalho permitiu definir características gerais dos pacientes de Alzheimer da cidade o que pode guiar estratégias de intervenção sobre estes pacientes na cidade.
\end{abstract}

RESUMO

PALAVRAS-CHAVE: Doença de Alzheimer, demência, Idoso.

\section{CLINICAL AND SOCIAL ASPECTS OF CAREGIVERS AND PEOPLE WITH ALZHEIMER'S DISEASE IN THE CITY OF MURITIBA-BA}

\section{ABSTRACT}

Alzheimer's disiease frequently affects the elderly, affecting cognitive functions such as memory, learning, language, attention, visual ability and spatial notion. In the city of Muritiba- BA, there is an increase in cases of Alzheimer's disease, so it was necessary to deepen the theme in the city to improve the quality of life of patients. The present study aimed to analyze the profile of patients with Alzheimer's disease in the city of Muritiba- BA in 2019. This is a descriptive cross-sectional study, with the 
population of patients with Alzheimer's disease in the city of Muritiba-BA, registered in the primary care of the municipality There was a higher prevalence of elderly people with Alzheimer's disease among females, of older age, with low education and income, and who were usually cared for by their daughter. Most elderly people used four or more medications, with quetiapine and donepezil being the most used. Most patients use more than 4 medications daily to fight disease and other illnesses. Thus, this work allowed the definition of general characteristics of Alzheimer's patients in the city, which can guide intervention strategies for these patients in the city.

KEYWORDS: Alzheimer Disease, dementia, elderly

\section{INTRODUÇÃO}

O crescente envelhecimento populacional nas últimas décadas traz como consequência um maior número de pessoas afetadas por doenças crônicas, dentre as quais se destaca a doença de Alzheimer, um importante problema de saúde pública da atualidade (MANZINI; VALE, 2020).

A Doença de Alzheimer é o tipo de doença cognitiva comum em idosos, afetando funções como a linguagem, memória, aprendizado, atenção, habilidade visual e noção espacial (NOROOZIAN , 2016). A Organização Mundial da Saúde (OMS) estima que 50 milhões de pessoas sofrem de algum tipo de demência no mundo, sendo $70 \%$ destes casos associados a doença de Alzheimer. No Brasil aproximadamente 1,2 milhões de pessoas são portadoras da doença de Alzheimer, e existe uma projeção de que este número triplique até o ano de 2050 (WHO, 2019).

O Alzheimer foi descrito pela primeira vez no ano de 1907 pelo psiquiatra alemão Alois Alzheimer e deste então, a doença continua impulsionando estudos para sua compreensão e busca por métodos efetivos de tratamento. Ainda existem muitas dúvidas sobre o tema, não somente dos portadores da doença e cuidadores, como também, de médicos e profissionais de saúde em geral (RINGMAN, 2017). Estas dúvidas se devem ao fato de que o Alzheimer pode ser confundido com outros tipos de demências comuns a pessoas idosas, ou mesmo com outras doenças degenerativas fazendo com que a doença apresente um difícil diagnóstico (CAIXETA, 2012).

O tratamento na maioria das vezes é composto por fármacos que atenuam os sintomas a nível cognitivo e funcional, como por exemplo o donepezil, a rivastigmina e a galatamina, contudo, pode haver intervenções não farmacológicas associadas ao tratamento, como o estimulo constante com atividades mentais e físicas, atividades de socialização, exercícios de memorização e tarefas domésticas no intuito de melhorar a qualidade de vida dos pacientes e facilitar o trabalho dos cuidadores (CARVALHO et al., 2016).

À medida que a doença evolui a pessoa idosa passa a depender de cuidados contínuos, realizados, na maioria dos casos, por um membro familiar no domicílio ou com o apoio de um cuidador formal contratado para auxiliar o indivíduo em tarefas domésticas e a utilização dos medicamentos (TALMELLI et al., 2013). O ato de cuidar de um paciente da doença de Alzheimer é uma atividade de grande complexidade, os familiares envolvidos nesta tarefa podem sofrer danos emocionais, físicos, problemas financeiros, distanciamento e abandono dos demais membros da família, impactando, diretamente, sobre os momentos de descanso, vida social e vida afetiva (ILHA et al., 2016, FALCÃO et al.,2018).

Muritiba é uma cidade do recôncavo baiano com uma população estimada em torno de 30.000 habitantes (IBGE,2020). A cidade tem aproximadamente $8 \%$ de sua população acima de 65 anos (IBGE,2020) e um dos problemas de saúde pública no 
município é a doença de Alzheimer, desta forma, se faz necessário o aprofundamento do tema no município através de divulgação científicas como esta, que podem servir de base para treinamentos dos médicos, profissionais de saúde do município, e população em geral para que o maior acesso a informação sobre as características da doença facilite outros diagnósticos e viabilize tratamentos farmacológicos e não farmacológicos mais assertivos, buscando uma melhoria da qualidade de vida do paciente, como também, ações que visem apoiar os cuidadores.

Sendo assim, esse trabalho teve como objetivo realizar um levantamento do número de pacientes com a Doença de Alzheimer município de Muritiba-BA e descrever o perfil dos pacientes portadores da doença, no intuito de coletar informações que possam guiar intervenções como campanhas informativas sobre a doença, visando melhorias no cuidado dos pacientes atuais e de novos pacientes no futuro.

\section{MATERIAIS E MÉTODOS}

Trata-se de um estudo descritivo de corte transversal com uma abordagem qualitativa e quantitativa. A população de estudo foi composta por pacientes com a Doença de Alzheimer, de ambos os sexos, cadastrados na atenção básica na cidade de Muritiba-Ba.

Muritiba é cidade localizada no recôncavo com população em torno de 30.000 habitantes, sendo aproximadamente $8 \%$ desta população com idade acima de 65 anos (IBGE, 2020). A cidade possui nove unidades de atenção básica de saúde da família, encontram-se cadastrados 17 pacientes portadores da doença de Alzheimer.

O critério de inclusão adotado para pesquisa foi ser portador da doença de Alzheimer, cadastrados na atenção básica de saúde do município. Como critério de exclusão apenas aqueles pacientes que não desejassem responder os questionários realizados pelos autores do trabalho.

A pesquisa foi realizada empregando como instrumento de coleta de dados um questionário criado pelos autores deste manuscrito, com perguntas objetivas e abertas, direcionado a busca de informações gerais sobre o paciente. Nas situações que o paciente não se encontrava em condições de responder isto era feito pelo respectivo cuidador.

O questionário foi composto por 8 perguntas, divididas em três categorias: dados sociais e demográficos do paciente (idade, gênero do paciente, parentesco do cuidador, estado civil, escolaridade, e renda mensal), dados do cuidador (gênero e parentesco com o paciente) e dados sobre diagnóstico e tratamento da patologia: tempo em que foi diagnosticada a doença e quais medicamentos utilizados. A coleta de dados ocorreu entre os meses de outubro e novembro de 2019, por entrevistadores treinados, nas residências dos pacientes, com o acompanhamento dos agentes comunitários e enfermeiras das unidades básicas de saúde.

A análise dos dados foi realizada através da utilização de planilhas do Excel o que permitiu a análise e associação com valores de frequência relativa. Cada tipo de resposta foi associada ao valor total da população alvo descrevendo os valores percentuais das características avaliadas, sendo expressas na forma de gráficos.

Esta pesquisa foi aprovada através do parecer no: 3.602 .224 pelo Comitê de Ética em Pesquisa da Faculdade Maria Milza-FAMAM/BA, respeitando as normas de pesquisa do Conselho Nacional de Saúde, conforme dispõe a Resolução CNS no 466/12 e Resolução CNS no 580/18, por se tratar de pesquisas envolvendo seres humanos. 


\section{RESULTADOS E DISCUSSÃO}

De posse dos dados coletados foi possível verificar que a amostra apresentou uma maioria de pacientes do sexo feminino (76\%) Figura 1, com média etária de 74,6 anos, sendo a maior concentração na faixa etária de 68 a 80 anos $(51,7 \%)$, com idade mínima geralmente de 66 e máxima de 88 anos.

FIGURA 1. Percentual de pacientes por gênero

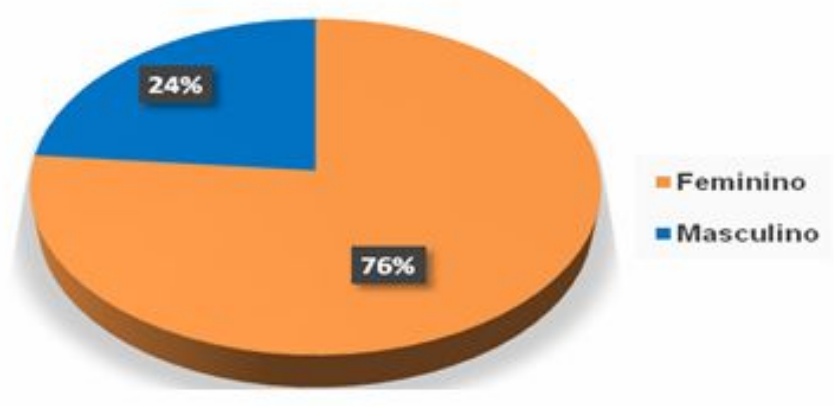

Fonte: Os Autores (2019)

O estudo revelou que $94 \%$ dos pacientes tinham filhos (Figura 2) e dentro dessa estimativa $61 \%$ dos cuidadores eram os próprios filhos (Figura 3), apenas $5 \%$ tratam-se de cuidadores profissionais que são supervisionados por um membro da família, compatível com o resultado de Martins et al., (2019), onde 65,4\% dos cuidadores eram os filhos.

FIGURA 2. Pacientes que possuem filhos.

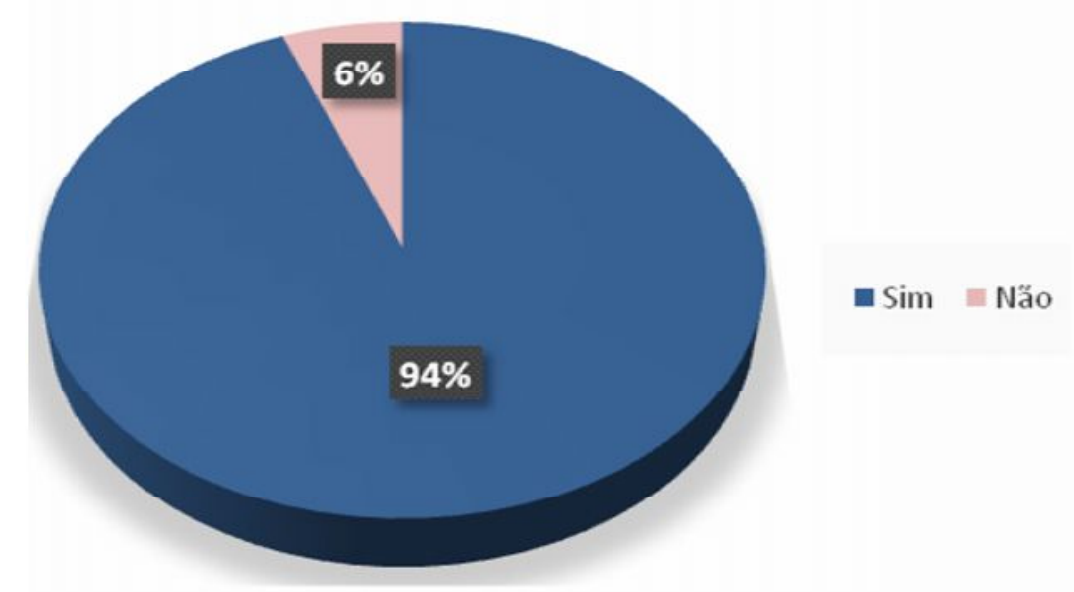

Fonte: Os Autores (2019) 
FIGURA 3. Grau de parentesco dos cuidadores

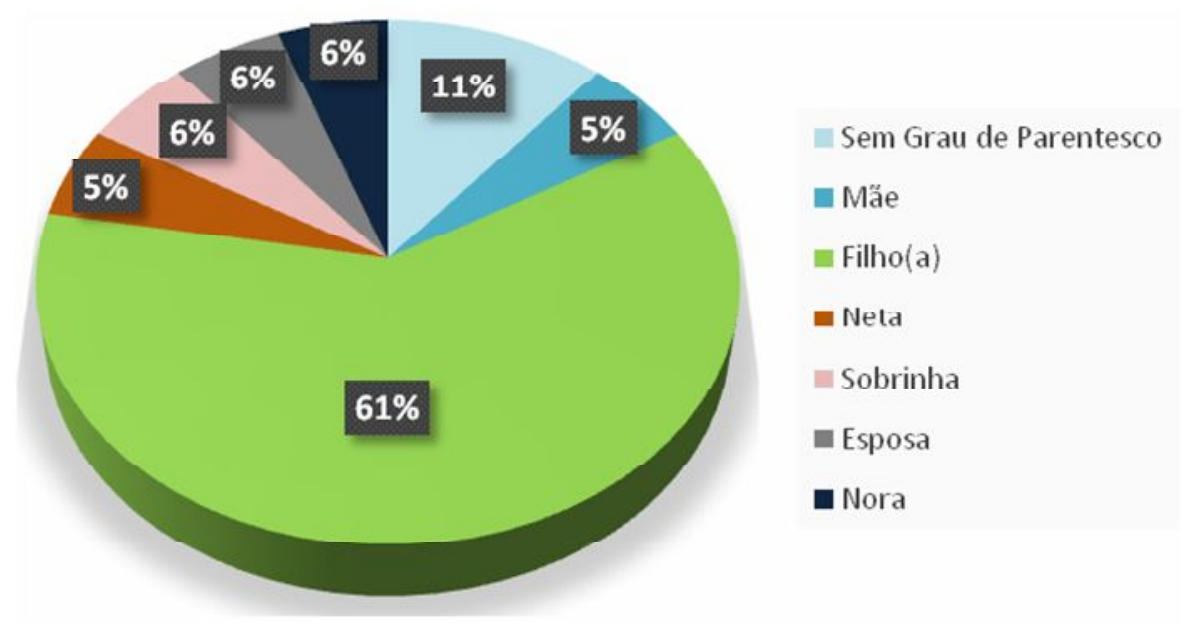

Fonte: Os Autores (2019)

Em relação aos cuidadores a amostra foi caracterizada por uma maioria feminina (94\%) (Figura 4), Concordando com trabalhos executados em outras cidades, como o feito por Martins et al.(2019) que mostrou que 96,2 \% dos cuidadores informais entrevistados eram do gênero feminino e metade destas eram casadas, e a pesquisa realizada por Seima et al., (2011) onde $86 \%$ dos cuidadores eram mulheres, sendo $61 \%$ casadas, isto demonstra a associação da mulher com a tarefa do cuidado e exclusão do homem neste processo.

FIGURA 4. Percentual de cuidadores por gênero.

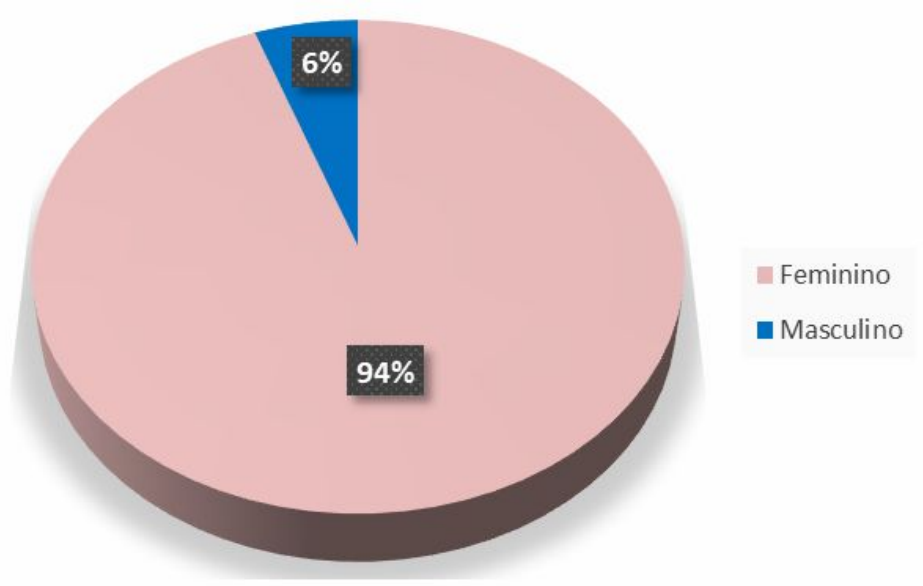

Fonte: Os Autores (2019)

Na pesquisa realizada por Araújo et al. (2013), 100\% dos cuidadores eram do sexo feminino, os autores justificam que apesar das mudanças recentes da sociedade brasileira, a figura masculina está geralmente associada ao trabalho fora do lar, provendo o sustento de sua família. Este dado é compatível com os resultados encontrados pela pesquisa realizada na cidade de Muritiba onde a maioria dos cuidadores é do sexo feminino.

A renda familiar média da maioria dos pacientes (Figura 5) varia em torno de 1 a 2 salários mínimos, este resultado sofre influência do fato de que a maioria dos 
idosos no Brasil, assim como os indivíduos participantes são aposentados e recebem esta faixa do benefício de aposentadoria. Esta renda baixa pode exercer influência na qualidade de vida dos pacientes uma vez que a escassez de recursos financeiros pode limitar o acesso das pessoas aos cuidados alimentares e sociais, dificultando o acesso a necessidades básicas do paciente, como os remédios, fraldas e terapias não farmacológicas (SOUZA et al., 2020).

FIGURA 5. Renda familiar média dos pacientes

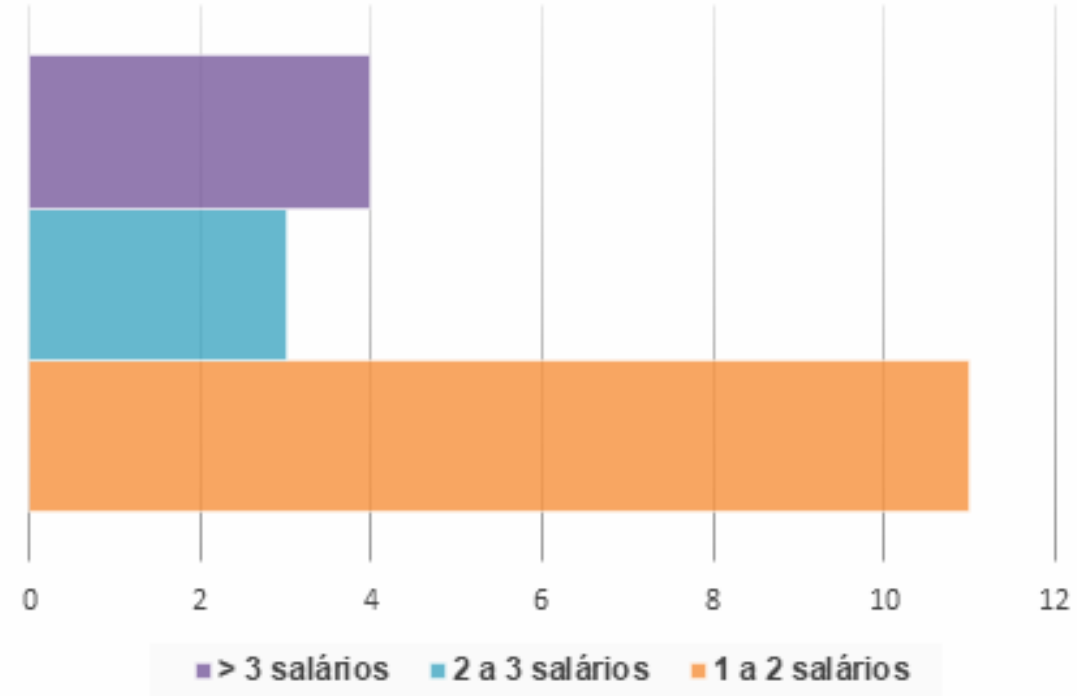

Fonte: Os Autores (2019)

Mesmo famílias que tem um pouco mais de condições financeiras relatam queixas sobre as dificuldades de manter os cuidados referentes ao paciente com doença de Alzheimer, em função dos custos com plano de saúde, contratação de profissionais para auxiliar nas tarefas diárias, consultas médicas particulares, medicamentos e fraldas geriátricas (MENDES; SANTOS 2016). A análise da escolaridade dos portadores da Doença de Alzheimer na cidade de Muritiba (Figura 6 ) demonstrou que a maioria dos pacientes (41\%), cursaram apenas o ensino fundamental incompleto.

FIGURA 6. Grau de escolaridade dos portadores da Doença de Alzheimer

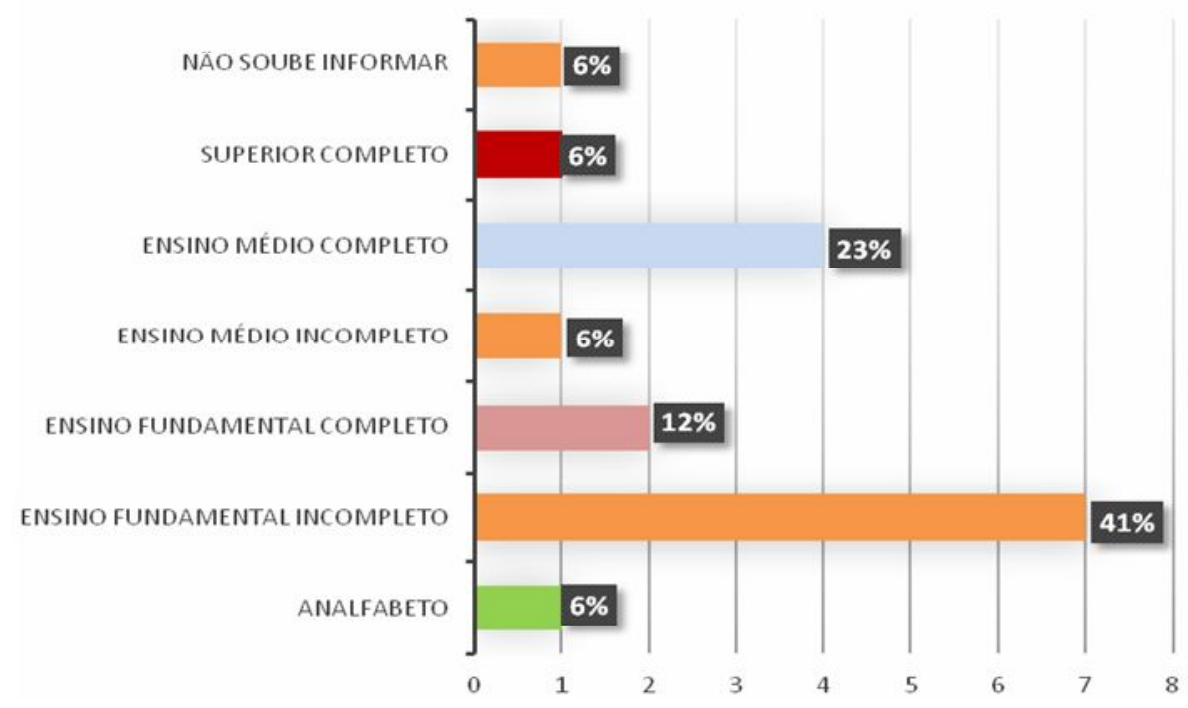

Fonte: Os Autores (2019) 
O estudo de Barroso et al. (2020), detectou que os pacientes com alta escolaridade tinham um desempenho melhor nos testes cognitivos do que os de baixa escolaridade. A literatura mostra resultados similares, creditando a escolaridade um fator protetor para perdas cognitivas e, consequentemente, menor comprometimento da capacidade funcional, quanto maior a atividade intelectual, menor a probabilidade de desenvolver a doença de Alzheimer, e menor o comprometimento cognitivo dos indivíduos acometidos com a doença (MELO; CADER 2018).

Além do déficit cognitivo, outras alterações de comportamento podem surgir. Neste estudo foi verificado que a maioria (59\%) dos idosos apresentam o comportamento brando, sugerindo apatia, 23\% costumam ter comportamentos variados, como fuga da realidade manifestadas no ato de dançar e cantar com frequência fora do normal, e em um quantitativo inferior apresentam comportamentos de agressividade (18\%), com dificuldade na realização das atividades básicas de vida diária, como o ato de tomar banho e se alimentar.

FIGURA 7. Comportamento dos pacientes com Doença de Alzheimer.

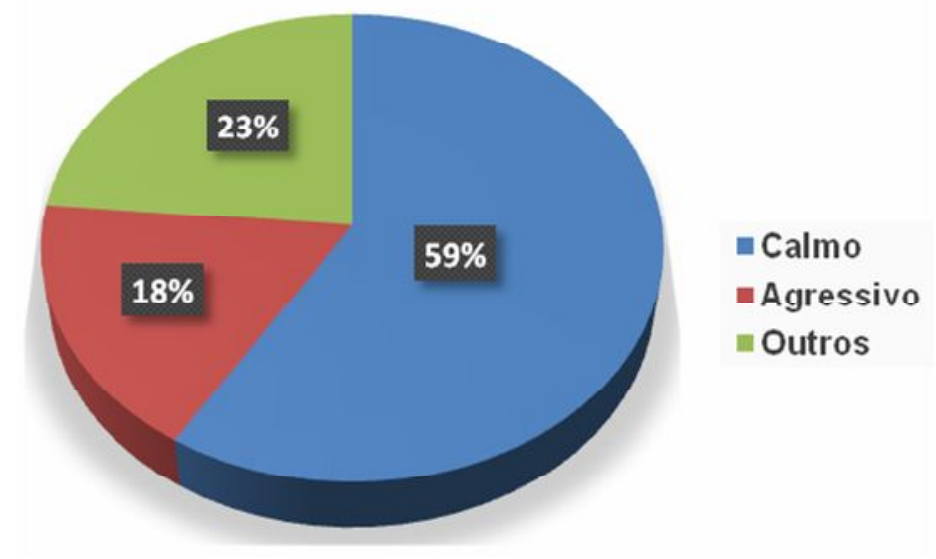

Fonte: Os Autores (2019)

Estes dados confirmam o que se conhece dos sintomas da doença, pois além da perda progressiva de memória específica e da função cognitiva, as manifestações da doença de Alzheimer frequentemente incluem distúrbios comportamentais, como apatia, agressividade e depressão ( JING et al., 2016; SILVA et al., 2019).

Este estudo também avaliou o tempo de diagnóstico da doença, como descrito no gráfico abaixo, a maior média em relação ao tempo de diagnóstico (Figura 8) foi maior que sete anos e $18 \%$ dos entrevistados não souberam informar. Mais de $50 \%$ dos pacientes em níveis diferentes da doença apresentaram um tempo de diagnóstico abaixo de cinco anos. 
FIGURA 8. Tempo de diagnóstico da Doença de Alzheimer.

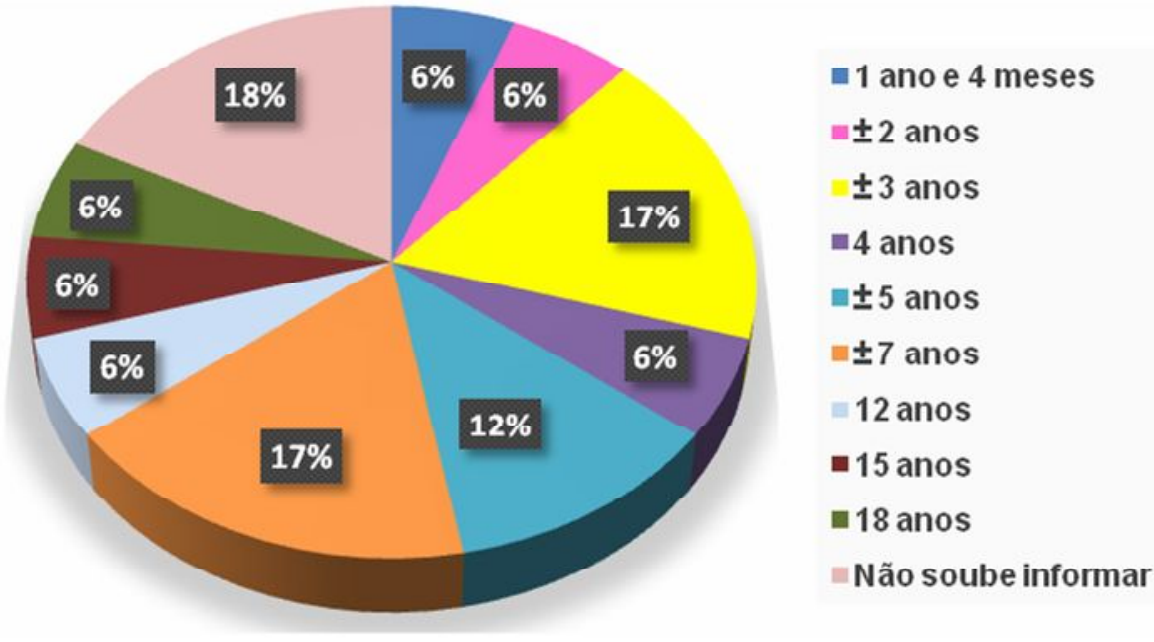

Fonte: Os Autores (2019)

O diagnóstico da doença de Alzheimer, em geral, é tardio e complexo devido a patologia ser confundida com outros tipos de demência, o que traz dificuldades para os pacientes ao longo do tratamento afetando a qualidade de vida. O diagnóstico na fase inicial da doença é importante para a redução da sua progressão e para garantir ao paciente, seus cuidadores e familiares bem-estar e qualidade de vida (DIAMOND et al., 2008). Desta forma é preciso destinar esforços para otimizar e antecipar os diagnósticos dos pacientes.

O tratamento da Doença de Alzheimer é executado pela associação de medicamentos com intervenções psicossociais para o paciente. Os inibidores da colinesterase, como o donepezil, galantamina e rivastigmina são os fármacos de primeira escolha para o tratamento da doença. Também podem ser utilizados na terapia antipiscóticos atípicos como a risperidona e a quetiapina, e o agente glutaminérgico memantina (BULCK et al., 2019).

A pesquisa permitiu uma avaliação da quantidade de medicamentos utilizados pelos indivíduos acometidos com a doença de Alzheimer na cidade. Cerca de $41 \%$ dos pacientes entrevistados faziam o uso de até dois medicamentos considerando não somente os empregados para o tratamento da doença e 54\% dos indivíduos usam a partir de quatro medicamentos diários sendo, portanto, considerados pacientes polifármacos (Figura 10).

FIGURA 10. Quantidade de remédios administrados por dia.

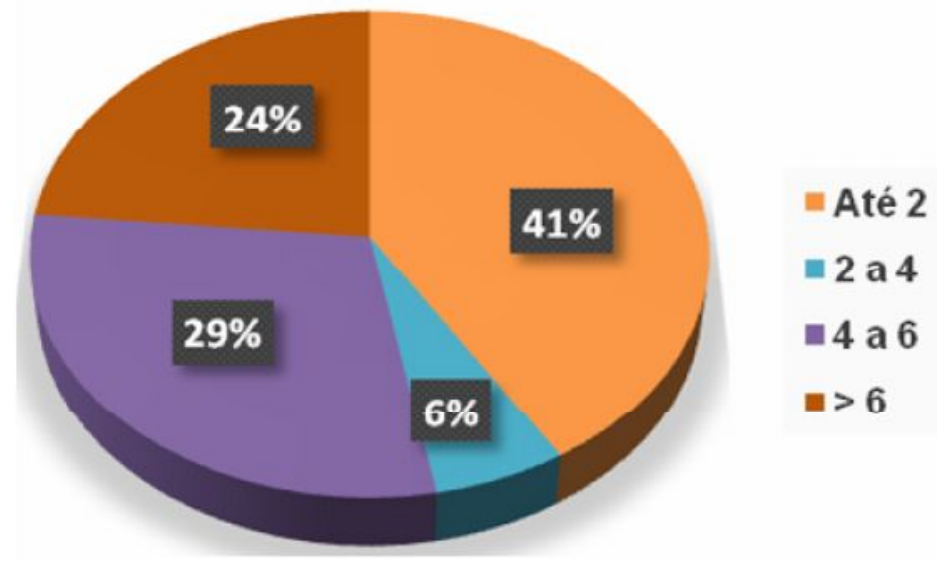

Fonte: Os Autores (2019) 
O uso elevado de medicamentos está associado ao fato do tratamento da doença Alzheimer frequentemente ser realizado através de associações de alguns fármacos, como os inibidores de colinesterase, antipisicóticos, antioxidantes ou antiinflamatórios. É necessário também considerar o fato de os pacientes em função da idade avançada apresentarem mais patologias fazendo uso de outros medicamentos além dos específicos para a terapia da doença de Alzheimer (SOUZA, 2018). O consumo de uma quantidade elevada de medicamentos gera problemas para a adesão total ao tratamento em função da elevação dos custos e quantidades de produtos diferentes para serem consumidos, aumenta os riscos à saúde devido a toxicidade cumulativa associada as interações medicamentosas entre os princípios ativos utilizados (ANDERSEN, 2011).

A literatura de forma geral mostra uma maior adesão a polifarmácia nos pacientes idosos, o trabalho de Souza et al. (2018) descreve a faixa de $92 \%$, enquanto Pinheiro et al. (2013) observaram a polifarmácia aderida por $73,3 \%$ dos pacientes, o resultado relativamente mais baixo dos pacientes da cidade de Muritiba $(54 \%)$ é diferenciado e pode indicar uma melhor qualidade de vida e saúde destas pessoas, a verificação das condições de vida destes indivíduos e dos tratamentos empregados podem auxiliar aos profissionais de saúde em busca de processos que favoreçam a redução da polifarmácia de pacientes em outras localidades.

Dentre as opções de tratamento entre os pacientes entrevistados, os medicamentos mais utilizados foram a donepezila e a quetiapina, (figura 9), medicamentos disponibilizados pelo sistema único de Saúde. Apesar da quetiapina não agir diretamente no suposto mecanismo de ação da doença, ela é um antipisicótico muito prescrito pelos médicos. Há evidências de que os antipsicóticos atuam no tratamento dos sintomas comportamentais frequentes nas síndromes demenciais, especialmente nos quadros de ansiedade e alterações do comportamento motor do paciente, amenizando os sintomas da doença (FORLENZA et al., 2008).

FIGURA 9. Percentual de medicamentos mais utilizados pelos pacientes.

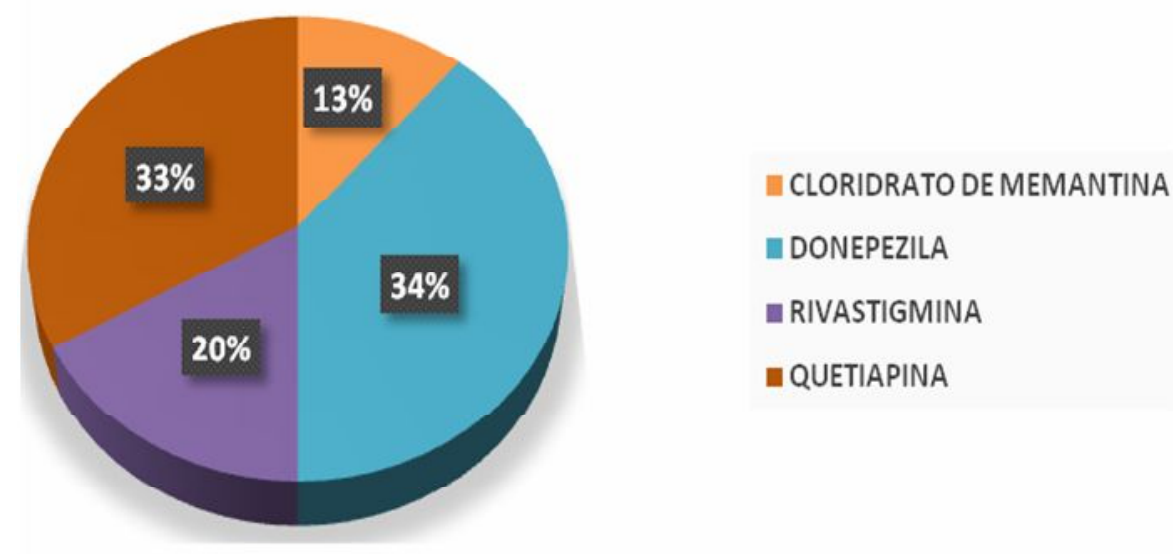

Fonte: Os Autores (2019)

\section{CONCLUSÃO}

Em função da elevação da expectativa de vida da população, as doenças relativas a terceira idade têm mostrado grande incidência. A doença de Alzheimer é uma das patologias cognitivas de maior prevalência entre pacientes idosos, o que faz merecer uma atenção especial com o intuito de melhorar a qualidade de vida dos indivíduos acometidos. 
A análise do perfil de pessoas com doença de Alzheimer na cidade de Muritiba, permitiu verificar que a maioria dos pacientes cadastrados na atenção básica de saúde do município apresentam perfil compatível com o descrito na literatura. Os pacientes em sua maioria são mulheres, com idade avançada apresentando baixa renda e escolaridade. Frequentemente estes pacientes são cuidados por filhos do sexo feminino prevalecendo ainda a forma tradicional de funcionamento familiar onde a mulher é quem assume as maiores responsabilidades de cuidar do idoso doente.

As informações obtidas por este estudo podem servir como base para o planejamento de estratégias que promovam auxílios de acesso a informações, cuidados ou terapias visando a melhoria de qualidade de vida para os pacientes e sua família.

\section{REFERÊNCIAS}

ANDERSEN, F.; VIITANEN, M.; HALVORSEN, D.S.; STRAUNE, B.; ENGSTAD, T.A. Co-morbidity and drug treatment in Alzheimer's disease. A cross sectional study of participants in the Dementia Study in Northern Norway. BMC Geriatrics. v.11, n.58, p. 1-7 Disponível em< https://pubmed.ncbi.nlm.nih.gov/21970467/> doi: 10.1186/1471-2318-11-58

ARAUJO, J.S.; VIDAL, G. M; BRITO, F. N; GONÇALVES, D. C.A; LEITE, D.K.M./ DUTRA, C. D. T.; PIRES, C. A.A. Perfil dos cuidadores e as dificuldades enfrentadas no cuidado ao idoso, em Ananindeua, PA. Revista Brasileira de Geriatria e Gerontologia, v. 16, n. 1, p. 149-158, 2013. Disponível em < https://www.scielo.br/pdf/rbgg/v16n1/a15v16n1.pdf> doi:10.1590/S180998232013000100015

BARROSO, M.C.R.D.; PESSOA, M.S.; PIRES P.A.P.; NETTO, A.C.M.G. Avaliação do Impacto do Nível Educacional na Doença de Alzheimer: Artigo Original. Revista Médica de Minas Gerais. v.30, p.1-4, 2020. Disponível em < http://www.rmmg.org/artigo/detalhes/2656>. doi: 10.5935/2238-3182.20200017

BULCK, S; SIERRA, A; ALARCON, J; PEREZ, A. C; MORALES, J. A. G. Novel Approaches for the Treatment of Alzheimer's and Parkinson's Disease. International Journal of Molecular Sciences. v. 20, n.3, 1-36 2019. Disponível em < https://www.mdpi.com/1422-0067/20/3/719>. doi:org/10.3390/jms20030719

CAIXETA, L. Evolução do conceito de doença de Alzheimer. In: Caixeta L (Org.). Doença de Alzheimer. Porto Alegre: Artmed; 2012.

CARVALHO, P.D.P.; MAGALHÃES, C.M.C.; PEDROSO, J.S. Tratamentos não farmacológicos que melhoram a qualidade de vida de idosos com doença de Alzheimer: uma revisão sistemática. Jornal Brasileiro de Psiquiatria. v. 65, n. 4, p. 334-339, 2016. Disponível em < https://www.scielo.br/scielo.php?pid=S004720852016000400334\&script=sci_abstract\&tlng=pt>. doi: 10.1590/00472085000000142

FALCÃO, D.; BRAZ, M.; GARCIA, C.; SANTOS, G.; YASSUDA, M.; CACHIONI, M.; NUNES, P.; FORLENZA, O. Atenção psicogerontológica aos cuidadores familiares de idosos com doença de Alzheimer. Psicologia, Saúde \& Doenças. v. 19, n.2, p. 
377-389,

2018.

Disponível

em<

http://www.scielo.mec.pt/pdf/psd/v19n2/v19n2a17.pdf>. doi: 10.15309/18psd190217

FORLENZA, O. V.; CRETAZ,E.; DINIZ, B,S.O.; O uso de antipsicóticos em pacientes

com diagnóstico de demência. Revista Brasileira de Psiquiatria. v. 30, n. 3, p. 265270, 2008. Disponível em < https://www.scielo.br/pdf/rbp/v30n3/a14v30n3.pdf >. doi: $10.1590 /$ S1516-44462008000300014

IBGE- Instituto Brasileiro de Geografia e Estatistica -Cidades | Bahia | Muritiba». cidades.ibge.gov.br. Consultado em 31 de agosto de 2020. Disponível em< https://cidades.ibge.gov.br/brasil/ba/muritiba/panorama $>$.

ILHA, S.; BACKES, D.S.; SANTOS, S.S.C.; GAUTÉRIO-ABREU, D.P.; SILVA, B.T.; PELZER, M.T. Doença de Alzheimer na pessoa idosa/família: Dificuldades vivenciadas e estratégias de cuidado. Escola Anna Nery. v.20, n.1, 2016. Disponível em<https://www.scielo.br/scielo.php?script=sci_arttext\&pid=S1414-

81452016000100138> doi: 10.5935/1414-8145.20160019

JING W.; WILLIS, R.; FENG, Z. Factors influencing quality of life of elderly people with dementia and care implications: A systematic review. Archives of Gerontology and Geriatrics, v. 66, p. 23-41, $2016 . \quad$ Disponível em < https://pubmed.ncbi.nlm.nih.gov/27176488/>. doi: 10.1016/j.archger.2016.04.009

MANZINI, C.S. S.; VALE, F.A.C. Transtornos emocionais evidenciados por cuidadores familiares de idosos com doença de Alzheimer. Dementia \& Neuropsychologia. v. 14, n.1, p. 56-61, 2020. Disponível em < https://www.scielo.br/scielo.php?pid=S1980-

57642020000100056\&script=sci_abstract\&tlng=pt>. doi:10.1590/198057642020dn14-010009

MARTINS, G.; CORRÊA, L.; CAPARROL, A. J. S.; SANTOS, P. T. A.; BRUGNERA, L. M; GRATÃO, A.C.M. Características sociodemográficas e de saúde de cuidadores formais e informais de idosos com Doença de Alzheimer. Escola Anna Nery. v. 23, n.2, p. 1-10, 2019. Disponível em < https://www.scielo.br/pdf/ean/v23n2/pt_14148145-ean-23-02-e20180327.pdf> doi: 10.1590/2177-9465-EAN-2018-0327

MENDES, C. F.M.; SANTOS, A.L.S. O cuidado na doença de Alzheimer: as representações sociais dos cuidadores familiares. Saúde e Sociedade. v. 25, n.1, p. 121-132, 2016.2 Disponível em < https://www.scielo.br/scielo.php?script=sci_arttext\&pid=S0104$12902016000100121 \&$ Ing=pt\&tlng=pt>. doi:10.1590/S0104-12902015142591.

MELO, M.; CADER, S.A. Caracterização e correlação do estado mental e da capacidade funcional de idosos asilados com mal de Alzheimer no Brasil e Paraguai. Revista Brasileira de Neurologia e Psiquiatria. v.22, n.2, p. 110-123, 2018. Disponível em<https://www.revneuropsiq.com.br/rbnp/article/view/270/152 >.

NOROOZIAN, M. Alzheimer's Disease: Prototype of Cognitive Deterioration, Valuable Lessons to Understand Human Cognition. Neurologic Clinics. v.34, n,1, 
p.69-131, 2016. Disponível em < https://europepmc.org/article/med/26613996> doi: 10.1016/j.ncl.2015.08.005

PINHEIRO, J.S.; CARVALHO, M.F.C.; LUPI, G. Interação medicamentosa e a farmacoterapia de pacientes geriátricos com síndrome demenciais. Revista Brasileira de Geriatria e Gerontologia. v.16, n.2, 303-314, 2013. Disponível em <https://www.scielo.br/pdf/rbgg/v16n2/10.pdf>. doi: 10.1590/S180998232013000200010

RINGMAN, J. M. Update on Alzheimer's and the Dementias. Neurologic Clinics. v.35, n.2, p. 171-174, 2017. Disponível em < https://pubmed.ncbi.nlm.nih.gov/28410654/> doi: 10.1016/j.ncl.2017.01.009

SEIMA, M.D.; LENARDT, M.H. A sobrecarga do cuidador familiar de idoso com Alzheimer. Textos \& Contextos (Porto Alegre), v.10, n.2, p.388-398, 2011.Disponível em

https://revistaseletronicas.pucrs.br/ojs/index.php/fass/article/view/9901/7341 >.

SILVA, M.V.F. ; LOURES, C.M.G.; ALVEZ, L.C.V.; SOUZA, L.C.; BORGES, K.B.G.; CARVALHO, M.G. Alzheimer's disease: risk factors and potentially protective measures. Journal of Biomedical Science. v. 26, n. 1, p.33-44, 2019. Disponível em < https://pubmed.ncbi.nlm.nih.gov/31072403/>. doi: 10.1186/s12929-019-0524-y

SOUZA, P,M.; KUSANO, L.T.E.; NETO, L.L.S. Prevalência da polifarmácia quantitativa e qualitativa em idosos com demência de Alzheimer. Geriatrics, Gerontology and Aging, v. 12, n. 3, p. 143-147, 2018. Disponível em < https://pesquisa.bvsalud.org/portal/resource/pt/biblio-948284>. doi: 10.5327/Z2447211520181800036

SOUZA, D.P.; MARTINS, K., P. A.; GOMES, H.; JESUS, A. G.; FIGUEIREDO, M. M. J.; SILVA, W. R. S.; FIGUEIREDO, K. M. J.; FILHO, E. S. S.; LIMA, P.R. Relação entre a qualidade de vida dos cuidadores de pacientes com doença de Alzheimer com aspectos socioeconômicos familiares e a gravidade da doença. Revista Eletrônica Acervo Saúde. v.12, n.4, p.1-10. 2020. Disponível em <https://acervomais.com.br/index.php/saude/article/view/879> doi: 10.25248/reas.e879.2020

TALMELLI, L. F.S.; VALE, F.A.C.; GRATÃO.A.C.M.; KUSUMOTA, L.; RODRIGUES, R.A.P. Doença de Alzheimer: declínio funcional e estágio da demência. Acta Paulista de Enfermagem. v. 26, n. 3, p. 219-225, 2013. Disponível em < https://www.scielo.br/scielo.php?script=sci_arttext\&pid=S0103-

21002013000300003\&lng=en > doi: 10.1590/S0103-21002013000300003

WHO - WORLD HEALTH ORGANIZATION. Alzheimer's Disease International; Dementia: Geneva: WHO; 2019. Disponível em https://www.who.int/news-room/factsheets/detail/dementia. Acessado em 09 de abril de 2020. 